\title{
Analysis of a chemostat model with variable yield coefficient: Contois kinetics
}

\author{
Rubayyi Turki Alqahtani ${ }^{1} \quad$ Mark I. Nelson ${ }^{2}$ \\ Annette L. Worthy ${ }^{3}$
}

(Received 8 January 2012; revised 15 May 2012)

\begin{abstract}
Food processing wastewaters and slurries typically contain high concentrations of biodegradable organic matter. Before the wastewater can be discharged, the pollutant concentration must be reduced. One way to achieve this is by using a biological species (biomass) that consumes the organic matter (substrate). We investigate an unstructured kinetic model for a bioreactor with a variable yield coefficient, taking into account the death rate of the microorganisms. The growth rate is given by a Contois expression, which is often used to model the growth of biomass in wastewaters containing biodegradable organic materials. The analysis shows that the system has natural oscillations for some ranges of the parameters. We also investigate the effects of the death rate parameter on the region of periodic behaviour.
\end{abstract}

http://journal.austms.org.au/ojs/index.php/ANZIAMJ/article/view/5093 gives this article, (c) Austral. Mathematical Soc. 2012. Published May 28, 2012. ISSN 1446-8735. (Print two pages per sheet of paper.) Copies of this article must not be made otherwise available on the internet; instead link directly to this URL for this article. 


\section{Contents}

1 Introduction

C156

1.1 The dimensional model . . . . . . . . . . . . . . . . C158

1.2 The dimensionless model . . . . . . . . . . . . . . . C159

2 Results

C160

2.1 Steady state solution branches . . . . . . . . . . C160

2.1.1 Stability along the washout branch . . . . . . . C162

2.1.2 Hopf bifurcation on the no-washout state . . . . . C162

2.2 Numerical results . . . . . . . . . . . . . . . . . . . C164

3 Conclusion

C169

References

C169

\section{Introduction}

Many industrial processes, particularly in the food industry, produce slurries or wastewaters containing high concentrations of biodegradable organic materials. Before these contaminated wastewaters can be discharged, the concentrations of pollutants must be reduced. Discharge to sewer may be possible but expensive, discharge to the environment would breach COD limits. A method which has been extensively employed to remove biodegradable organic matter is biological treatment. In this process, the wastewater (or slurry) is passed through a bioreactor containing biomass which grows by consuming the pollutants. The growth of the biomass produces more biomass and other products, a mixture of carbon dioxide, methane, water and biological compounds.

Many mathematical models for the biological treatment of wastewater use Monod kinetics. However, extensive experimental work has shown that the 
anaerobic and aerobic degradation of wastewater originating from industrial processes is often better described by the Contois growth rate expression [1, $2,3,4,5,6]$. Thus, in this work the degradation of a biodegradable organic material is represented by the Contois growth rate expression [3].

Only a few mathematical studies have used the Contois model to simulate the biological treatment of wastewater [1, 2]. Nelson and Sidhu [2] analysed a well-mixed continuously stirred bioreactor using a Contois expression with a variable yield coefficient. They found that there are regions in the parameter space in which a Hopf bifurcation occurs. Ajbar et al. [1] extended this model to take into account oxygen transfer limitations. Neither of these studies $[1,2]$ considered the death rate of the microorganisms.

We study the behaviour of a generalised reactor model that encompasses a continuous flow bioreactor and an idealised continuous flow membrane reactor as limiting cases with microorganism death rate. The specific growth rate of the biomass is assumed to be given by a Contois expression [3] with a variable yield coefficient.

We find the steady state solutions, determine their stability, and obtain asymptotic solutions in the limit of high residence times. We determine the conditions for washout to occur and the regions of parameter values for which periodic behaviour can be generated.

The main contributions of this study are the following.

1. Studying the effect of the death rate with a variable yield coefficient on the bioreactor.

2. Studying a generalised reactor model, including a continuous flow bioreactor and an idealised continuous flow membrane reactor as limiting cases. 


\subsection{The dimensional model}

The model equations are

$$
\begin{aligned}
& V \frac{d S}{d t}=F\left(S_{0}-S\right)-V X \frac{\mu(S, X)}{Y(S)}, \\
& V \frac{d X}{d t}=-F \beta X+V X \mu(S, X)-K_{d} V X
\end{aligned}
$$

for the specific growth rate (Contois models)

$$
\mu(S, X)=\mu_{m}\left(\frac{S}{K_{s} X+S}\right)
$$

residence time

$$
\tau=\frac{V}{F}
$$

and yield coefficient

$$
Y(S)=\alpha+\gamma S, \quad(\alpha, \gamma>0)
$$

The units that the concentrations of the substrate species, $\mathrm{S}$, and the microorganisms, $X$, are measured in are denoted by $|S|$ and $|X|$ respectively. The parameters in the model are:

- $F$, the flow rate through the reactor $\left(\mathrm{dm}^{3} \mathrm{day}^{-1}\right)$;

- $\mathrm{K}_{\mathrm{d}}$, the death coefficient $\left(\right.$ day $\left.^{-1}\right)$;

- $\mathrm{K}_{\mathrm{s}}$, the saturation constant $\left(|\mathrm{X}||\mathrm{S}|^{-1}\right)$;

- $S$, the substrate concentration within the reactor $(|S|)$;

- $S_{0}$, the concentration of substrate flowing into the reactor $(|S|)$;

- $\mathrm{V}$, the volume of the reactor $\left(\mathrm{dm}^{3}\right)$; 
- $\mathrm{X}$, the microorganism concentration within the reactor $(|\mathrm{X}|)$;

- $t$, the time (day);

- $\alpha$, the yield factor $\left(|X||S|^{-1}\right)$;

- $\gamma$, a constant in the yield coefficient $\left(|X||S|^{-2}\right)$;

- $\mu(S, X)$, the specific growth rate model $\left(\right.$ day $\left.^{-1}\right)$;

- $\mu_{\mathrm{m}}$, the specific growth rate parameter $\left(\right.$ day $\left.^{-1}\right)$; and

- $\tau$, the liquid residence time (day).

The parameter $\beta$ defines the reactor model for the reactor. The choice $\beta=1$ gives a continuous flow reactor whereas $\beta=0$ gives an idealised membrane reactor, in which all of the microorganisms are forced to remain in the reactor vessel. The choice $0<\beta<1$ gives a non-idealised membrane reactor. In this case some of the microorganisms leave the reactor vessel while some stay in the reactor vessel. For a non-idealised membrane reactor to be operationally effective we require $0<\beta \ll 1$. Most modern membranes are very close to ideal, the intermediate values of $\beta$ represent a settler rather than a membrane. It is assumed that the operating environment variables such as the temperature and $\mathrm{pH}$ are held constant.

\subsection{The dimensionless model}

The system of differential equations $(1,2)$ are written in dimensionless form by introducing dimensionless variables for the substrate concentration $S^{*}=S / S_{0}$, the microorganism concentration $X^{*}=K_{s} X / S_{0}$, and time $t^{*}=\mu_{m} t$,

$$
\begin{aligned}
\frac{d S^{*}}{d t^{*}} & =\frac{1}{\tau^{*}}\left(1-S^{*}\right)-\frac{S^{*} X^{*}}{\left(\alpha^{*}+\gamma^{*} S^{*}\right)\left(S^{*}+X^{*}\right)}, \\
\frac{d X^{*}}{d t^{*}} & =\frac{-\beta X^{*}}{\tau^{*}}+\frac{S^{*} X^{*}}{S^{*}+X^{*}}-K_{d}^{*} X^{*} .
\end{aligned}
$$


In Equations $(6,7), \mathrm{K}_{\mathrm{d}}^{*}=\mathrm{K}_{\mathrm{d}} / \mu_{\mathrm{m}}$ is the dimensionless death rate, $\alpha^{*}=\mathrm{K}_{\mathrm{s}} \alpha$ is the dimensionless yield coefficient, $\tau^{*}=\mathrm{V} \mu_{\mathrm{m}} / \mathrm{F}$ is the dimensionless liquid residence time, and $\gamma^{*}=\gamma \mathrm{K}_{\mathrm{s}} \mathrm{S}_{0}$ is a dimensionless constant in the yield coefficient. In Equations $(6,7)$ the main experimental control parameter is the dimensionless residence time $\tau^{*}$.

We investigate this model with the assumptions that: the concentration of substrate entering into the reactor and the death rate are both positive, $S_{0}>0$, $\mathrm{K}_{\mathrm{d}}^{*}>0$. We assume in the following that $0<\mathrm{K}_{\mathrm{d}}^{*}<1$.

\section{Results}

In Section 2.1, we show that there are two physically meaningful steady state solution branches. These are a washout branch and a no-washout branch. In Section 2.1.1, the stability of the washout solution is determined. The Hopf bifurcation on the no-washout state is discussed in Section 2.1.2. In Section 2.2 , the steady state diagrams are presented.

The XPP [7] software was used to obtain the steady state diagrams. The standard representation is: solid lines are stable steady states; dotted lines are unstable steady states; filled-in squares are Hopf bifurcation points; open circles are unstable periodic orbits; and filled-in circles are stable periodic solutions.

\subsection{Steady state solution branches}

The steady state solutions of the system of equations (6) and (7) are the following.

- The washout branch:

$$
\left(S^{*}, X^{*}\right)=(1,0) .
$$


- The no-washout branches:

$$
\begin{aligned}
& \left(S^{*}, X^{*}\right)=\left(\widehat{S}^{*}, \frac{\left(\alpha^{*}+\gamma^{*} \widehat{S}^{*}\right)\left(1-\widehat{S}^{*}\right)}{\beta+\tau^{*} K_{d}^{*}}\right), \\
& \widehat{S}^{*}=\frac{-b}{2 a}+\frac{\sqrt{b^{2}-4 a c}}{2 a} \\
& a \widehat{S}^{* 2}+b \widehat{S}^{*}+c=0,
\end{aligned}
$$

Where $a, b$, and $c$ are defined by

$$
a=\gamma^{*}, \quad b=\left(1-K_{d}^{*}\right) \tau^{*}+\alpha^{*}-\beta-\gamma^{*}, \quad c=-\alpha^{*} .
$$

Note that the coefficient $\boldsymbol{a}$ is strictly positive whereas $c$ is strictly negative. This means that the solution (7) for $\mathbf{S}^{*}$ is always positive. From Equation (9) the steady state solution is positive (physically meaningful) when $0<\mathrm{S}^{*}<1$. The residence time at which we have $S^{*}=1$ is

$$
\tau^{*}=\tau_{\mathrm{cr}}^{*}=\frac{\beta}{1-\mathrm{K}_{\mathrm{d}}^{*}} .
$$

Thus, the no-washout steady state is physically meaningful when the residence time satisfies

$$
\tau^{*}>\tau_{\mathrm{cr}}^{*}=\frac{\beta}{1-\mathrm{K}_{\mathrm{d}}^{*}} .
$$

Implicity differentiating Equation (11), we obtain,

$$
\frac{d S^{*}}{d \tau^{*}}=\frac{\left(1-K_{d}^{*}\right) S^{*}}{-2 a S^{*}-b}<0 \text { because } S^{*}>\frac{-b}{2 a} .
$$

Thus the steady state effluent concentration is a decreasing function of the residence time. At large residence times, the approximate value of the microorganism concentration and substrate concentration are

$$
\begin{aligned}
& \mathrm{X}^{*} \approx \frac{\alpha^{*}}{\mathrm{~K}_{\mathrm{d}}^{*} \tau^{*}}+\mathrm{O}\left(\frac{1}{\tau^{* 2}}\right), \quad 0<\mathrm{K}_{\mathrm{d}}^{*}<1, \\
& \mathrm{~S}^{*} \approx \frac{\alpha^{*}}{\left(1-\mathrm{K}_{\mathrm{d}}^{*}\right) \tau^{*}}+\mathrm{O}\left(\frac{1}{\tau^{* 2}}\right), \quad 0<\mathrm{K}_{\mathrm{d}}^{*}<1 .
\end{aligned}
$$




\subsubsection{Stability along the washout branch}

Under washout conditions the effluent concentration is equal to the inflow concentration. Thus this state of operation must be avoided. The eigenvalues of the Jacobian matrix at the washout state are

$$
\lambda_{1}=-\frac{1}{\tau^{*}}<0, \quad \lambda_{2}=\frac{-\beta+\tau^{*}\left(1-K_{d}^{*}\right)}{\tau^{*}} .
$$

Hence, the washout branch is stable when

$$
\tau^{*}<\frac{\beta}{1-\mathrm{K}_{\mathrm{d}}^{*}} .
$$

Equation (17) shows that a perfect membrane cannot have washout, a good membrane could only have washout at a very small residence time.

\subsubsection{Hopf bifurcation on the no-washout state}

In this section we investigate the stability of the no-washout state by evaluating the Jacobian matrix in the no-washout state. We find

$$
\mathrm{J}\left(\mathrm{S}^{*}, \mathrm{X}^{*}\right)=\left[\begin{array}{ll}
\mathrm{J}_{11} & \mathrm{~J}_{12} \\
\mathrm{~J}_{21} & \mathrm{~J}_{22}
\end{array}\right],
$$

where

$$
\begin{aligned}
& \mathrm{J}_{11}=-\frac{1}{\tau^{*}}+\frac{\mathrm{X}^{*}\left(\gamma^{*} \mathrm{~S}^{* 2}-\alpha^{*} \mathrm{X}^{*}\right)}{\left(\alpha^{*}+\gamma^{*} \mathrm{~S}^{*}\right)^{2}\left(\mathrm{X}^{*}+\mathrm{S}^{*}\right)^{2}}, \quad \mathrm{~J}_{22}=-\frac{\mathrm{X}^{*} \mathrm{~S}^{*}}{\left(\mathrm{X}^{*}+\mathrm{S}^{*}\right)^{2}}, \\
& \mathrm{~J}_{21}=\frac{\mathrm{X}^{*^{2}}}{\left(\mathrm{X}^{*}+\mathrm{S}^{*}\right)^{2}}, \quad \mathrm{~J}_{12}=-\frac{\mathrm{S}^{*^{2}}}{\left(\alpha^{*}+\gamma^{*} \mathrm{~S}^{*}\right)\left(\mathrm{X}^{*}+\mathrm{S}^{*}\right)^{2}} .
\end{aligned}
$$

The conditions for both eigenvalues to be zero are $\mathrm{J}_{11} \mathrm{~J}_{22}-\mathrm{J}_{21} \mathrm{~J}_{12}=0$ and $\mathrm{J}_{11}+\mathrm{J}_{22}=0$. Some algebra shows that the first condition cannot be satisfied 
for a physically meaningful solution and as a result a double zero eigenvalue degeneracy cannot occur.

The physical significance of a Hopf bifurcation is that it is one of the two main mechanisms by which periodic behaviour is generated in systems of nonlinear differential equations, the other being a double zero eigenvalue bifurcation. The conditions for a Hopf bifurcation to occur are $J_{11} J_{22}-J_{21} J_{12}>0$ and $\mathrm{J}_{11}+\mathrm{J}_{22}=0$. The first condition is always satisfied. The values of the residence time at which Hopf bifurcations occur are found to satisfy the equations,

$$
\begin{aligned}
\psi & =r_{1} X^{*^{2}}+r_{2} X^{*}+r_{3}=0 \\
b_{1} & =\frac{\beta+K_{d}^{*} \tau^{*}}{\tau^{*}\left(1-K_{d}^{*}\right)-\beta}, \quad r_{1}=b_{1}{ }^{2} \gamma^{* 2}\left[\left(1+b_{1}\right)^{2}+\tau^{*} b_{1}\right], \\
r_{2} & =b_{1} \gamma^{*}\left[2 \alpha^{*}\left(1+b_{1}\right)^{2}+\tau^{*} b_{1}\left(2 \alpha^{*}-1\right)\right], \\
r_{3} & =\alpha^{*}\left[\alpha^{*}\left(1+b_{1}\right)^{2}+\tau^{*}\left(b_{1} \alpha^{*}+1\right)\right] .
\end{aligned}
$$

Note that when $\tau^{*}>\tau_{c r}^{*}$ then $b_{1}>0$ and hence $r_{1}$ and $r_{3}$ are positive.

From these equations, the following results are obtained.

1. When the yield coefficient is constant (that is, $\gamma=0$ ), the trace is negative and the Hopf bifurcation conditions (19) are not satisfied. Thus the variability of the yield coefficient is a significant condition for producing a natural oscillation. Under these conditions, the no-washout branch is always stable.

2. The coefficient $r_{2}$ is positive when $\alpha^{*} \geqslant 1 / 2$ and as result Equation (19) is the sum of positive terms and cannot have zero value. Thus the Hopf bifurcation cannot occur when

$$
\alpha^{*} \geqslant \frac{1}{2}
$$

3. For the membrane reactor $(\beta=0)$, the condition given in (19) simplifies 
considerably. After some algebra the solution of Equation (19) is

$$
X^{*}=\frac{-r_{2} \pm \sqrt{\tau^{*}\left(1-K_{d}^{*}\right)\left(K_{d}^{*}\left(1-K_{d}^{*}\right)\left(4 \alpha^{*}-K_{d}^{*}\right) \tau^{*}-4 \alpha^{*}\right)}}{2 r_{1}} .
$$

This solution is complex when $K_{d}^{*} \geqslant 4 \alpha^{*}$. Thus, Hopf bifurcations cannot occur when

$$
\mathrm{K}_{\mathrm{d}}^{*} \geqslant 4 \alpha^{*}
$$

A degenerate Hopf bifurcation is where two Hopf points annihilate each other in an unfolding diagram (a $\mathrm{H} 21$ degeneracy). The conditions for this to occur are

$$
\mathrm{J}_{11} \mathrm{~J}_{22}-\mathrm{J}_{21} \mathrm{~J}_{12}>0, \quad \psi=0, \quad \frac{\mathrm{d} \psi}{\mathrm{d} \tau^{*}}=0 .
$$

When $\alpha^{*}=0.01$ and $\mathrm{K}_{\mathrm{d}}^{*}=0.01$ the solutions that satisfy the $\mathrm{H} 2{ }_{1}$ degenerate conditions are

$$
\left(\gamma^{*}, \tau^{*}\right)=(13.328,14.741), \quad\left(\gamma^{*}, \tau^{*}\right)=(0.022,1.077) .
$$

Natural oscillations cannot occur in the regions $0.022>\gamma^{*}$ and $\gamma^{*}>13.328$. They do occur in the region $0.022<\gamma^{*}<13.328$.

\subsection{Numerical results}

Figure 1 shows two steady state diagrams. Each steady state diagram contains two curves for the washout $\left(S^{*}=1\right)$ and the no-washout states $\left(S^{*}<1\right)$. Our choice of the values of the parameter $\gamma^{*}$ in Figure 1 are motivated by the values in Equation (24). In Figure 1(a) there are two Hopf bifurcation points because the value of $\gamma^{*}$ is in the region $0.022<\gamma^{*}<13.328$. Between the two Hopf bifurcation points, the no-washout branch is unstable and there are stable periodic solutions. 


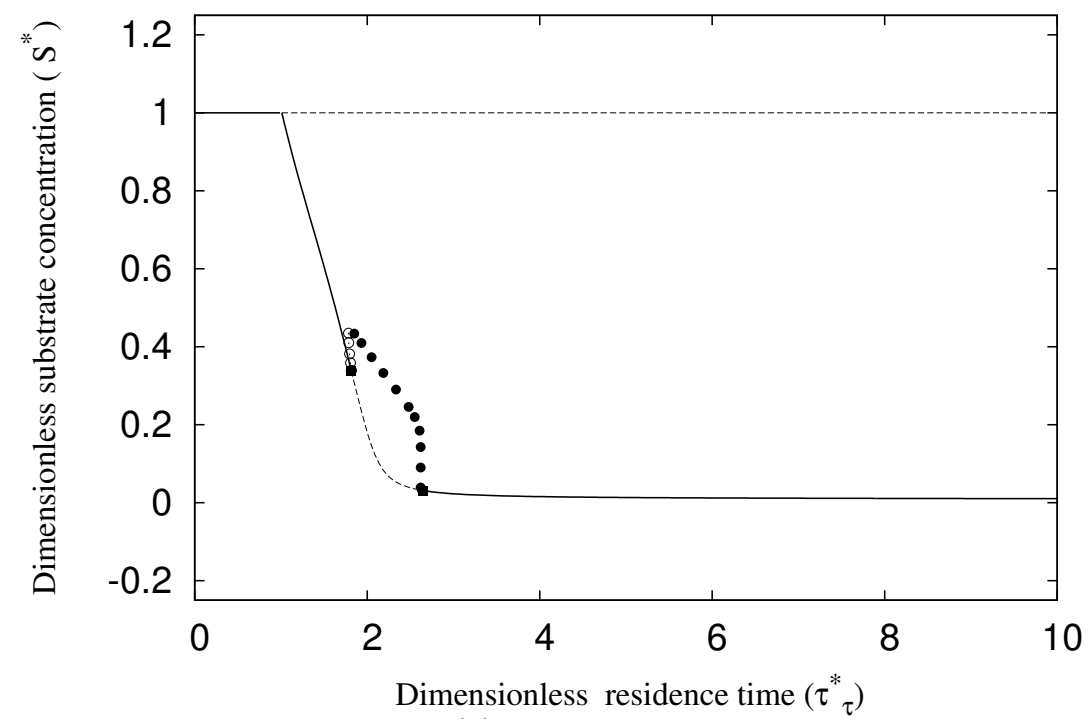

(a) $\gamma^{*}=1.05$

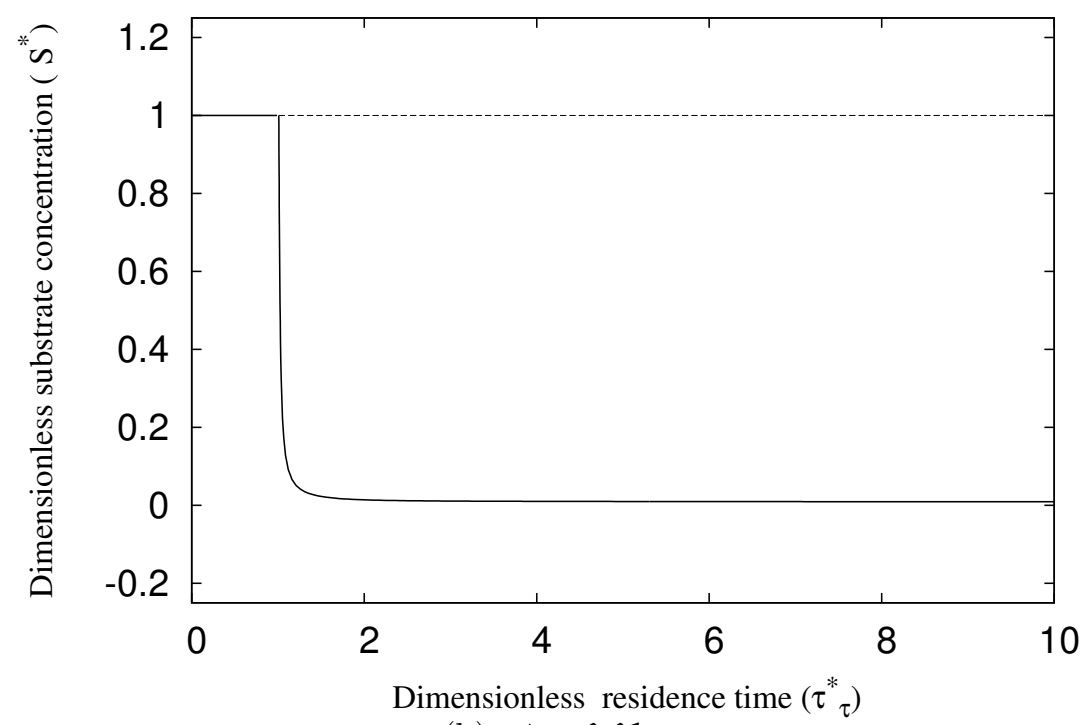

(b) $\gamma^{*}=0.01$

Figure 1: Dimensionless effluent concentrations as a function of the dimensionless residence time. Parameters $\beta=1, \mathrm{~K}_{\mathrm{d}}^{*}=0.01, \alpha^{*}=0.01$. 


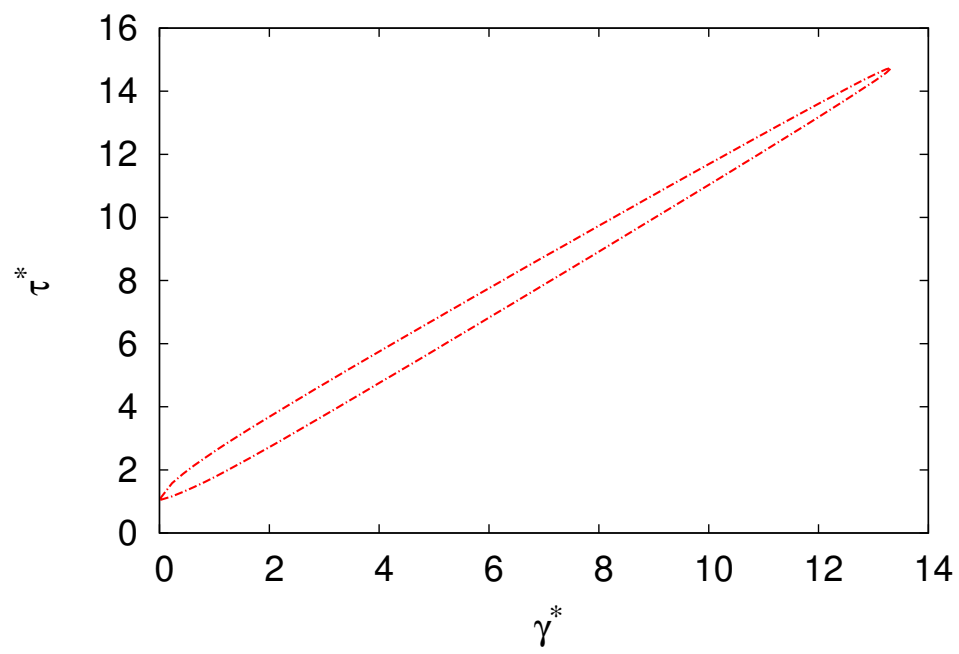

Figure 2: Unfolding diagram for a single reactor showing the Hopf bifurcation locus. Parameters $\beta=1, \mathrm{~K}_{\mathrm{d}}^{*}=0.01, \alpha^{*}=0.01$.

In Figure 1(b) there are no periodic solutions as the value of $\gamma^{*}$ is outside the critical region and the no-washout solution is always stable provided $\tau^{*}>\tau_{\mathrm{cr}}^{*}$. Figure 2 is an unfolding diagram for the two Hopf bifurcations in Figure 1(a) in the $\gamma^{*}-\tau^{*}$ plane. Figure 2 shows the values of the residence time at which a Hopf bifurcation occurs as a function of $\gamma^{*}$ : the Hopf bifurcation points are only found in the region $\left(0.022<\gamma^{*}<13.328\right)$ and they destroy each other at the $\mathrm{H} 2{ }_{1}$ degeneracy given by equation (24). In the region between the $\mathrm{H} 2_{1}$ degeneracies, periodic oscillations occur. For either large or small values of $\gamma^{*}$, including the constant yield case $\left(\gamma^{*}=0\right)$, periodic behaviour does not occur. The periodic solutions are undesirable in this system because the average effluent concentration of a periodic solution is higher than the corresponding value of the unstable steady state solution.

Figure 3 shows $\mathrm{H} 2{ }_{1}$ degeneracy curves, defined by (23), in the $\alpha^{*}-\gamma^{*}$ plane.

Figure 3(a) shows that there is a critical value of the death rate, $K_{d}^{*}=$ 0.2872 . If $0<\mathrm{K}_{\mathrm{d}}^{*}<0.2872$ then the $\mathrm{H} 2_{1}$ curve has two limit points $\left(\alpha_{1, \mathrm{Lp}}^{*}>\right.$ 


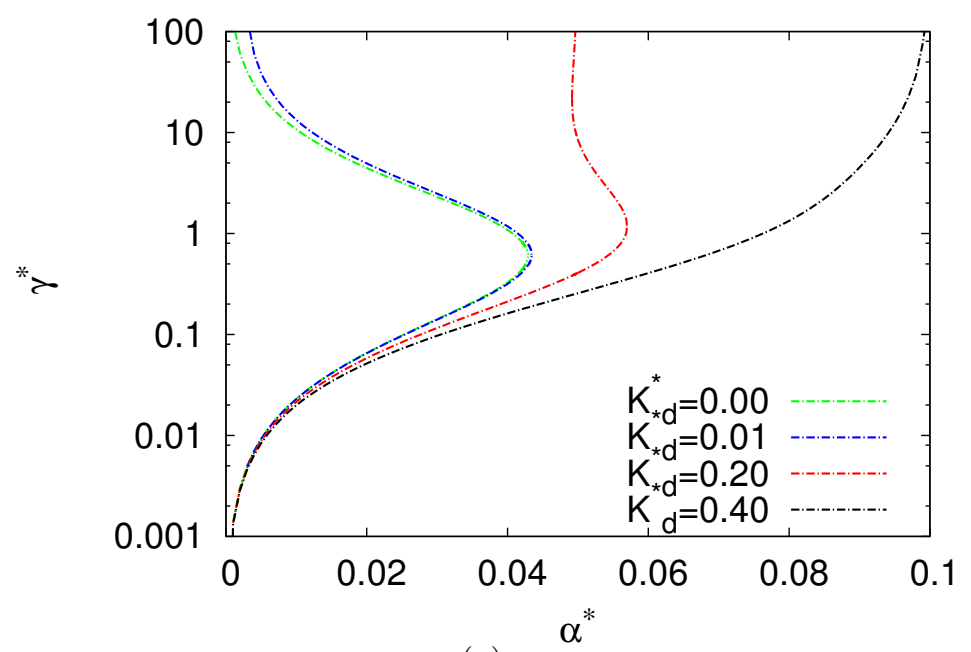

(a)

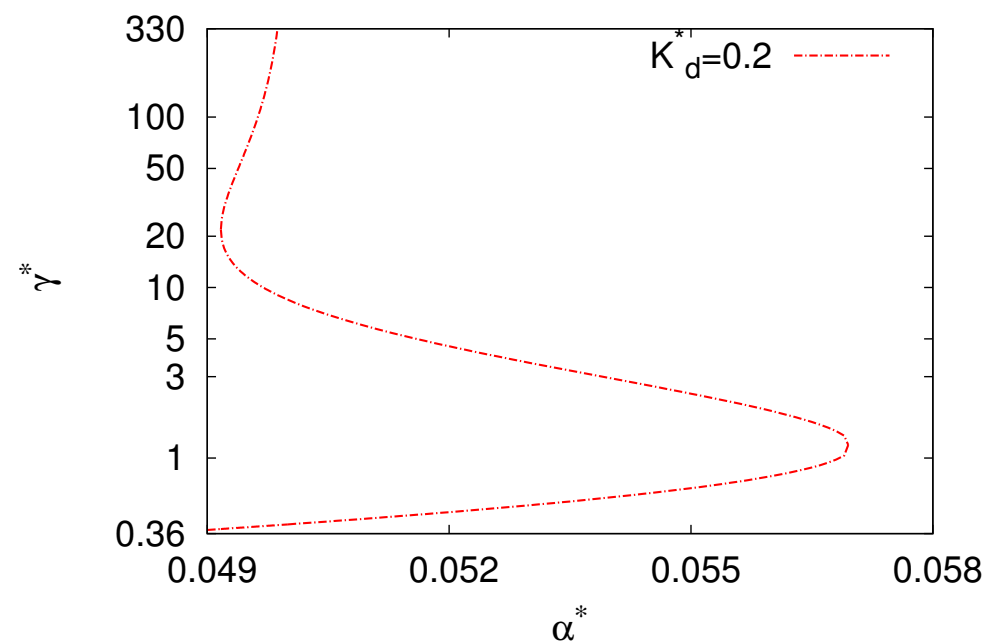

(b)

Figure 3: $\mathrm{H} 2{ }_{1}$ degeneracy diagram. Parameter $\beta=1$. 


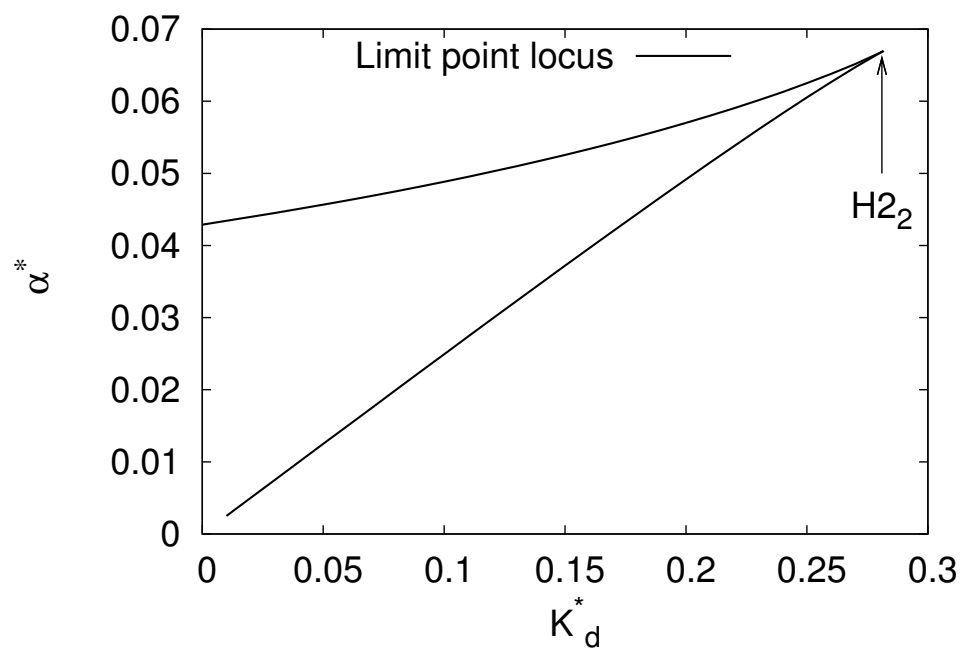

Figure 4: Diagram showing the domain of the limit point locus in parameter spaces $\left(K_{d}^{*}, \alpha^{*}\right)$. Parameter $\beta=1$.

$\alpha_{2, \mathrm{Lp}}^{*}$ ). Depending upon the value for $\alpha^{*}$ there may be no, one, two or three $\mathrm{H} 2{ }_{1}$ degeneracies. For example, Figure $3(\mathrm{~b})$ shows that when $\mathrm{K}_{\mathrm{d}}^{*}=0.2$ there are two limit points and accordingly, in the region between $0.049<\alpha^{*}<0.050$ there are three $\mathrm{H} 2_{1}$ degeneracies. When $0<\mathrm{K}_{\mathrm{d}}^{*}<0.2872$ there are no $\mathrm{H} 2_{1}$ points, and consequently no Hopf bifurcations, when the yield constant coefficient is sufficiently high, $\alpha^{*}>\alpha_{1, \mathrm{Lp}}^{*}$.

When $\mathrm{K}_{\mathrm{d}}^{*}>0.2872$ the $\mathrm{H} 2_{1}$ degeneracy curve has no limit points and there is always one $\mathrm{H} 2{ }_{1}$ degeneracy, see the curve on Figure $3(\mathrm{a})$ when $\mathrm{K}_{\mathrm{d}}^{*}=0.4$. If the value of $\gamma^{*}$ is below the $\mathrm{H} 2{ }_{1}$ degeneracy curve, then there are no Hopf bifurcation points on the steady state diagram. If it is above, then the steady state diagram contains two Hopf bifurcations.

Figure 4 summarises the information about limit points that is provided in Figure 3(a): the limit point $\alpha_{1, \mathrm{Lp}}^{*}>\alpha_{2, \mathrm{Lp}}^{*}$ is possible for all values of $\mathrm{K}_{\mathrm{d}}^{*}$ less than the critical value $\mathrm{K}_{\mathrm{d}}^{*}=0.2872$. 


\section{Conclusion}

We analysed the behaviour of a bioreactor using a Contois growth model with a variable yield coefficient. We extended earlier work [2] by including the death rates of microorganisms and by using a generalized bioreactor model which includes as special cases the continuous flow and membrane reactors.

We determined a critical value of the residence time at which the washout solution loses stability. This critical value is the minimum residence time that can be used. Our investigation of the reactor's periodic behaviour shows that there is a wide range of parameter values for which natural oscillations occur. The analysis of the effect of the death rate on the oscillatory behaviour shows that there is a critical value of the death rate $\left(\mathrm{K}_{\mathrm{d}}^{*}=0.2872\right)$. If the death rate is less than this critical value, then there may be between zero and three $\mathrm{H} 2{ }_{1}$ degeneracies. If the value of the death rate is higher than the critical value, then one $\mathrm{H} 2{ }_{1}$ degeneracy can occur. We have also shown that the range of the yield constant coefficient for which oscillations are possible increases for high values of the death rate.

We showed that in the region of periodic behaviour the stable periodic solution has a higher average effluent concentration than the unstable steady state solution and consequently the reactor performance decreases.

Acknowledgements RA thanks Dammam University for a PhD Scholarship, and thanks the CSIRO/ANZIAM Student Support Scheme.

\section{References}

[1] Ajbar, A., Al Ahmad, M. and Ali, E. (2010) On the dynamics of biodegradation of wastewater in aerated continuous bioreactors. 
Mathematical and Computer Modelling, 54 (9-10):1930-1942. doi:10.1016/j.mcm.2011.04.035 C157

[2] Nelson, M.I. and Sidhu, H.S., (2007). Reducing the emission of pollutants in food processing wastewaters. Chemical Engineering and Processing, 46 (5):429-436. doi:10.1016/j.cep.2006.04.012 C157, C169

[3] Contois, D.E. (1959). Kinetics of bacterial growth: relationship between population density and specific growth rate of continuous cultures. Journal of general microbiology, 21:40-50. doi:10.1099/00221287-21-1-40 C157

[4] Alqahtani, R. T., M. I. Nelson, and A. L. Worthy. (2012). A fundamental analysis of continuous flow bioreactor models with recycle around each reactor governed by Contois kinetics. III. Two and three reactor cascades. Chemical Engineering Journal 183 (0):422-432. doi:10.1016/j.cej.2011.12.061 C157

[5] Abdurahman, N. H., Y. M. Rosli, and N. H. Azhari. (2011). Development of a membrane anaerobic system (MAS) for palm oil mill effluent (POME) treatment. Desalination 266 (1-3):208-212. doi:10.1016/j.desal.2010.08.028 C157

[6] Hu, W.C., Thayanithy, K., Forster, C.F. (2002). A kinetic study of the anaerobic digestion of ice-cream wastewater. Process biochemistry, 37:965-971. doi:10.1016/S0032-9592(01)00310-7 C157

[7] Bard Ermentrout, (2002). Simulating, Analyzing, and Animating Dynamical Systems: A Guide to XPPAUT for Researchers and Students, Society for Industrial and Applied Mathematics. doi:10.1137/1.9780898718195 C160 


\section{Author addresses}

1. Rubayyi Turki Alqahtani, School of Mathematics and Applied Statistics, University of Wollongong, Wollongong, NSW 2522, Australia.

mailto:rtaa648@uowmail.edu.au

2. Mark I. Nelson, School of Mathematics and Applied Statistics, University of Wollongong, Wollongong, NSW 2522, AUstralia. mailto:mnelson@uow.edu. au

3. Annette L. Worthy, School of Mathematics and Applied Statistics, University of Wollongong, Wollongong, NSW 2522, AUstralia mailto:annette_worthy@uow.edu.au 